\title{
Dynamic heterogeneity in an orientational glass
}

\author{
Nirvana B. Caballero, ${ }^{1, a)}$ Mariano Zuriaga, ${ }^{2, b)}$ Josep Lluís Tamarit, ${ }^{3, c)}$ and Pablo Serra ${ }^{2, d)}$ \\ ${ }^{1}$ CNEA, CONICET, Centro Atomico Bariloche, 8400 San Carlos de Bariloche, Rio Negro, Argentina \\ ${ }^{2}$ Facultad de Matemática, Astronomía, Física y Computación, Universidad Nacional de Córdoba, Córdoba, \\ Argentina and IFEG-CONICET, Ciudad Universitaria, X5016LAE Córdoba, Argentina \\ ${ }^{3}$ Grup de Caracterització de Materials, Departament de Física, EEBE and Barcelona Research Center \\ in Multiscale Science and Engineering, Eduard Maristany, 10-14, 08019 Barcelona, Catalonia, Spain
}

(Received 14 September 2017; accepted 26 October 2017; published online 13 November 2017)

\begin{abstract}
The family of compounds $\mathrm{CBr}_{n} \mathrm{Cl}_{4-n}$ has been proven helpful in unraveling microscopic mechanisms responsible for glassy behavior. Some of the family members show translational ordered phases with minimal disorder which appears to reveal glassy features, thus deserving special attention in the search for universal glass anomalies. In this work, we studied $\mathrm{CBrCl}_{3}$ dynamics by performing extensive molecular dynamics simulations. Molecules of this compound perform reorientational discrete jumps, where the atoms exchange equivalent positions among each other revealing a cage-orientational jump motion fully comparable to the cage-rototranslational jump motion in supercooled liquids. Correlation times were calculated from rotational autocorrelation functions showing good agreement with previous reported dielectric results. From mean waiting and persistence times calculated directly from trajectory results, we are able to explain which microscopic mechanisms lead to characteristic times associated with $\alpha$ - and $\beta$-relaxation times measured experimentally. We found that two nonequivalent groups of molecules have a longer characteristic time than the other two nonequivalent groups, both of them belonging to the asymmetric unit of the monoclinic (C2/c) lattice. Published by AIP Publishing. https://doi.org/10.1063/1.5004671
\end{abstract}

\section{INTRODUCTION}

Glassy dynamics are present in all physical systems with non-stationary dynamical processes in observable time scales, where due to competitive interactions, the system remains trapped in metastable states. Such dynamical processes are usually present in systems with a large number of metastable configurations. At present, this behavior has been observed in quite diverse systems, like granular materials, ${ }^{1}$ biologic evolution models, ${ }^{2}$ elastic strings in disordered media, ${ }^{3}$ domain wall dynamics, ${ }^{4}$ and many substances and mixtures of substances of highly relevant technological applications. ${ }^{5}$

The glass and glass transition concepts were developed from the study of supercooled liquids. However, some of their properties were found in other condensed matter systems. In fact, detailed theoretical considerations of molecular liquids ${ }^{6}$ and some kind of molecular crystals ${ }^{7}$ have proven useful to establish connections with theoretical results derived from several statistical mechanics approaches where glassiness appears in the dynamics of idealized objects. Such model systems show trivial, non-interacting, equilibrium behaviors but nonetheless, interesting slow dynamics features appear due to restrictions on the allowed transition between configurations. ${ }^{8}$

Disordered molecular crystals known as rotator-phases or plastic-crystals are simple systems (commonly with cubic lattice symmetry) exhibiting dynamical behaviors which may at

\footnotetext{
a)Electronic mail: nirvana.caballero@cab.cnea.gov.ar

b)Electronic mail: zuriaga@famaf.unc.edu.ar

c)Electronic mail: josep.lluis.tamarit@upc.edu

d)Electronic mail: serra@famaf.unc.edu.ar
}

least partially be understood by recourse to results obtained from idealized models. ${ }^{9}$ In these kinds of systems, molecules may reorient by almost free rotations at the nodes of a crystal lattice and by cooling such a rotator phase, a frozen orientationally disordered crystalline state (called glassy crystal or orientational glass) may be attained, exhibiting fully developed glassy behavior. Moreover, thermodynamic signatures similar to those common to vitrifying liquids are shown when approaching this state. ${ }^{9-11}$

There is also the possibility that a phase transition to a lower symmetry crystal occurs, involving a partial reduction of the orientational disorder, but keeping some occupational (low-dimensional) disorder. These systems ${ }^{12-18}$ can still display glassy features and represent the most simplified models on which theoretical concepts on glassy dynamics can be tested. Materials composed by tetrahedral molecules of general formula $\mathrm{CBr}_{n} \mathrm{Cl}_{4-n}, n=0, \ldots, 4$, exhibit a series of solid-solid phase transitions with increasing temperature before melting attributed to the ability of the molecules to acquire rotational degrees of freedom as the temperature is increased. ${ }^{19-21}$ Cooling the room temperature liquids leads to rotationally disordered crystalline phases (rotator or plastic phase) which show translational face-centered cubic or rhombohedral lattices with the carbon atoms sitting at the lattice nodes. Further cooling leads to a transformation into complex monoclinic, $\mathrm{C} 2 / \mathrm{c}$ structures with $\mathrm{Z}=32$ molecules per unit cell and an asymmetric unit with $\mathrm{Z}^{\prime}=4 .{ }^{19-25}$

Molecular motions within such monoclinic crystals persist down to $\sim 90 \mathrm{~K}$ where a calorimetric transition much alike that exhibited by the canonical glass-transition signals the transition into a orientationally disordered state where 
molecular reorientations cannot be detected with the available experimental means. ${ }^{26}$ The dynamics of the monoclinic phases of $\mathrm{CBr}_{n} \mathrm{Cl}_{4-n}, \mathrm{n}=0,1,2$, compounds has been studied by means of dielectric spectroscopy and nuclear quadrupole resonance (NQR) spectroscopy ${ }^{27,28}$ in the temperature ranges $100-250 \mathrm{~K}$ and $80-210 \mathrm{~K}$, respectively. The former technique allows the measurement of the dynamic response within a broad time scale but it is insensitive to fine details of molecular motions, whereas the latter has a restricted time window but monitors the movement of individual chlorine atoms. Results derived from the concurrent use of NQR and molecular simulations show that large-angle rotations of tetrahedral about their higher symmetry axes $\left(\mathrm{CBrCl}_{3}\right.$ and $\mathrm{CBr}_{2} \mathrm{Cl}_{2}$ with $C_{3 v}$ and $C_{2 v}$ point-group symmetries, respectively) lead to a statistical occupancy of $75 \%$ for $\mathrm{Cl}$ and $25 \%$ for $\mathrm{Br}$ atoms in the case of $\mathrm{CBrCl}_{3}$ and $50 \%$ for $\mathrm{Cl}$ and $50 \%$ for $\mathrm{Br}$ atoms for $\mathrm{CBr}_{2} \mathrm{Cl}_{2}$ in agreement with $\mathrm{X}$-ray and neutron diffraction measurements. ${ }^{20,21,29,30}$ These experimental techniques also show that the relaxation movements arise from different dynamics exhibited by molecules in the asymmetric unit of the crystalline lattice that are non-equivalent with respect to their molecular environment.

The dielectric spectra of $\mathrm{CBrCl}_{3}$ and $\mathrm{CBr}_{2} \mathrm{Cl}_{2}$ at the lower end of the temperature range display a well-defined shoulder on the high frequency flank of the $\alpha$-peak, which is attributed to the $\beta$-relaxation. ${ }^{27}$ Since $\mathrm{CCl}_{4}$ has no molecular dipole moment, it is not accessible to dielectric experiments but it can be studied using NQR. Interestingly, the resolution of the NQR spectra for $\mathrm{CCl}_{4}$ is well superior to the corresponding spectra for $\mathrm{CBr}_{2} \mathrm{Cl}_{2}$ and $\mathrm{CBrCl}_{3}$ and the two techniques complement each other. On the other hand, the NQR experiments are limited to a temperature range between $77 \mathrm{~K}$ and $140 \mathrm{~K}$, with the upper end determined by the broadening of the signal. ${ }^{28}$ The picture that emerges from the combined analysis is that the three compounds have a very similar dynamic evolution in the monoclinic phase as a function of temperature. ${ }^{27,28}$ The analysis of isostructural $\mathrm{CCl}_{4}$ shows that nonequivalent molecules in the unit cell perform reorientational jumps at different time scales due to their different crystalline environments. These results support the conclusion that the dynamic heterogeneity is intimately related to the secondary relaxation observed in these compounds. . $^{27,28,31}$

The currently accepted scenario for canonical glasses includes different relaxation mechanisms that are universally present in all systems. Experimentally, these different mechanisms are enclosed into the dielectric spectra that show a broad low-frequency peak referred to as $\alpha$-relaxation ${ }^{32}$ and a higher frequency peak or shoulder usually called Johari-Goldstein $\beta$-relaxation. ${ }^{33-44}$ The $\alpha$-relaxation is due to processes involving cooperative dynamics of regions of molecules. ${ }^{32}$ The microscopic origin of the $\beta$ relaxation is still a matter of wide debate. ${ }^{33-36,38-40,42-45}$ Some of the proposed models explain this peak as a consequence of the non-uniformity of the glassy state involving only local regions in which molecules can diffuse (islands of mobility). An alternative homogeneous explanation attributes the secondary relaxation phenomena to small-angle reorientations of all molecules..$^{12,46,47}$

In our previous work on $\mathrm{CCl}_{4},{ }^{48}$ we found that the monoclinic phase of this compound has essentially the same dynamical behavior, as a function of temperature, as that of its isostructural glass formers, $\mathrm{CBrCl}_{3}$ and $\mathrm{CBr}_{2} \mathrm{Cl}_{2}$. The simulations clearly show that there are preferential axes of rotation, which are fixed with respect to the crystal orientation. Two of the inequivalent groups of molecules among the four nonequivalent molecules of the asymmetric unit are significantly faster than the other two, leading to a clear heterogeneity in the dynamics of the system. Moreover, it is found that the orientation of the two fast axes of rotation is the same, suggesting an overall dynamics anisotropy correlated to the molecular orientations. We showed that the different reorientational dynamics in $\mathrm{CCl}_{4}$ (not involving structural changes) are responsible for characteristic times compatible with $\alpha$ - and $\beta$-relaxation times, measured with dielectric spectroscopy for the members of the family which can orientationally vitrify.

In this work, we have studied the monoclinic phase of $\mathrm{CBrCl}_{3}$ using molecular dynamics simulations in order to elucidate the mechanisms responsible for the $\alpha$ - and $\beta$-relaxation observed experimentally. ${ }^{27,28}$

\section{THEORETICAL METHODS}

\section{A. Model and computational details}

$\mathrm{CBrCl}_{3}$ is a non-regular tetrahedral molecule with $C_{3 v}$ molecular symmetry. We have modeled $\mathrm{CBrCl}_{3}$ molecules as rigid, non-polarizable tetrahedra with the carbon atom at the center, three chlorine atoms on three vertices, and a bromine atom located in the remaining vertex, as was proposed in our previous work. ${ }^{49}$ The interaction between molecules is represented by a combination of Lennard-Jones and Coulombic terms summarized in Table I. The cross interaction between atoms of different types is calculated by applying the Lorentz-Berthelot combination rules, i.e., geometrical mean for $\epsilon$ and arithmetic mean for $\sigma$. A spherical cutoff at $1.8 \mathrm{~nm}$ was imposed on all intermolecular interactions. Periodic boundary conditions were imposed in all three Cartesian directions.

The isostructural series $\mathrm{CBr}_{n} \mathrm{Cl}_{4-n}$ have a lowtemperature monoclinic crystal structure, resolved by Cohen et al. at $195 \mathrm{~K}^{50}$ which corresponds to the $C 2 / c$ space group. The unit cell, with $Z=32$ molecules, has the following lattice parameters: $a=2.0631 \mathrm{~nm}, b=1.1619 \mathrm{~nm}, c=2.0201 \mathrm{~nm}$, and angle $\beta=111.19^{\circ}$ at $220.2 \mathrm{~K} .{ }^{51}$ Using the experimental crystalline structure as initial coordinates, we constructed a simulation super-cell containing 512 molecules, which correspond to 16 monoclinic unit cells. This super-cell was prepared by replicating the experimental unit cell twice on the $x$ and $z$ directions and four times in the $y$ direction.

The molecular dynamics simulations, conducted under NPT conditions, have been carried out using the Gromacs v5.0.2 simulation package. Atom-atom distances within each

TABLE I. $\mathrm{CBrCl}_{3}$ model parameters and geometry.

\begin{tabular}{lccrlr}
\hline \hline & $\epsilon(\mathrm{kJ} / \mathrm{mol})$ & $\sigma(\mathrm{nm})$ & \multicolumn{1}{c}{$q(\mathrm{e})$} & \multicolumn{2}{c}{ Bond $(\mathrm{nm})$} \\
\hline $\mathrm{C}$ & 0.22761 & 0.37739 & -0.696 & $\mathrm{C}-\mathrm{Cl}$ & 0.1766 \\
$\mathrm{Cl}$ & 1.09453 & 0.34667 & 0.174 & $\mathrm{Cl}-\mathrm{Cl}$ & 0.2884 \\
$\mathrm{Br}$ & 2.13000 & 0.37200 & 0.177 & $\mathrm{C}-\mathrm{Br}$ & 0.1944 \\
\hline \hline
\end{tabular}


molecule were kept constants with the SHAKE algorithm. The classical Newton's equations were integrated using the leap-frog algorithm and the time step of the integration of the equations of motion was set to $5 \mathrm{fs}$.

The production runs were extended up to $200 \mathrm{~ns}$ or $11 \mu \mathrm{s}$ depending on the temperature. The temperature control was implemented with a Nosé-Hoover thermostat, with a time constant of $2.0 \mathrm{ps}$. The pressure was maintained constant by using a fully anisotropic Parrinello-Rahman barostat with a reference pressure of $1 \mathrm{~atm}$. The study covered temperatures ranging from $160 \mathrm{~K}$ to $220 \mathrm{~K}$, in steps of $10 \mathrm{~K}$.

\section{RESULTS}

Molecular dynamics simulations of $\mathrm{CBrCl}_{3}$ for the whole range of studied temperatures capture the essential dynamics of $\mathrm{CBrCl}_{3}$ proposed to the moment: we observe a monoclinic structure, where carbon atoms are centered in the lattice nodes and all molecules perform reorientational jumps between equilibrium positions.

Molecular rotations were characterized by angular selfcorrelation functions defined as ${ }^{52}$

$$
C^{b}(t)=\frac{1}{N} \sum_{i}^{N}\left\langle\vec{u}_{i}^{b}(\zeta) \cdot \vec{u}_{i}^{b}(t+\zeta)\right\rangle_{\zeta},
$$

where $\vec{u}_{i}^{b}$ is the normalized vector directed along one of the $\mathrm{CBrCl}_{3}$ intra-molecular bonds $b=\mathrm{C}-\mathrm{Br}, \mathrm{C}-\mathrm{Cl}_{a}, \mathrm{C}-\mathrm{Cl}_{b}, \mathrm{C}-\mathrm{Cl}_{c}$, $i$ is the molecule number, and $N$ is the number of molecules considered in the calculation. The average is over times $\zeta$. These self-correlation functions were calculated for the four molecular bonds for all molecules in the system. We found the same behavior for all three $\mathrm{C}-\mathrm{Cl}$ bonds, but a slower dynamics for the $\mathrm{C}-\mathrm{Br}$ bonds. The functions are shown for bonds $b=\mathrm{C}-\mathrm{Br}$ and $b=\mathrm{C}-\mathrm{Cl}_{a}$ in Fig. 1 for the whole range of studied temperatures.

$\mathrm{CBrCl}_{3}$ is isostructural to $\mathrm{CCl}_{4}$. The $\mathrm{CCl}_{4}$ unit cell is monoclinic, with $\mathrm{Z}=32$ molecules per unit cell and space group $\mathrm{C} 2 / \mathrm{c}$. The spatial positions of the molecules in the unit cell are defined through the application of 8 symmetry operations over $\mathrm{Z}^{\prime}=4$ nonequivalent molecules. As a consequence, the system has four distinctive groups of molecules (according to the $\mathrm{Z}^{\prime}=4$ molecules in the asymmetric unit and $\mathrm{Z}=32$ molecules in the unit cell) that we will refer to as groups I, II, III, and IV. In our simulation cell, each group contains 128 molecules. In the $\mathrm{CCl}_{4}$ system, each molecule belonging to a given group has the same specific arrangement of neighboring molecules. However, in a $\mathrm{CBrCl}_{3}$ system, the bromine atoms introduce disorder in the system and molecules belonging to the same group have no longer the same environment.

In order to get deeper understanding of the system rotational dynamics, we have also calculated the self-correlation functions of molecules belonging to the four non-equivalent groups. These functions are shown in Fig. 2 for $\mathrm{T}=160 \mathrm{~K}$. We found that groups I and IV have similar dynamics, and the same conclusion holds for groups II and III, for all studied temperatures. Here, molecules in groups II and III have faster reorientational dynamics than molecules belonging to groups I and IV. The two different dynamics of nonequivalent molecules in the asymmetric unit were previously detected
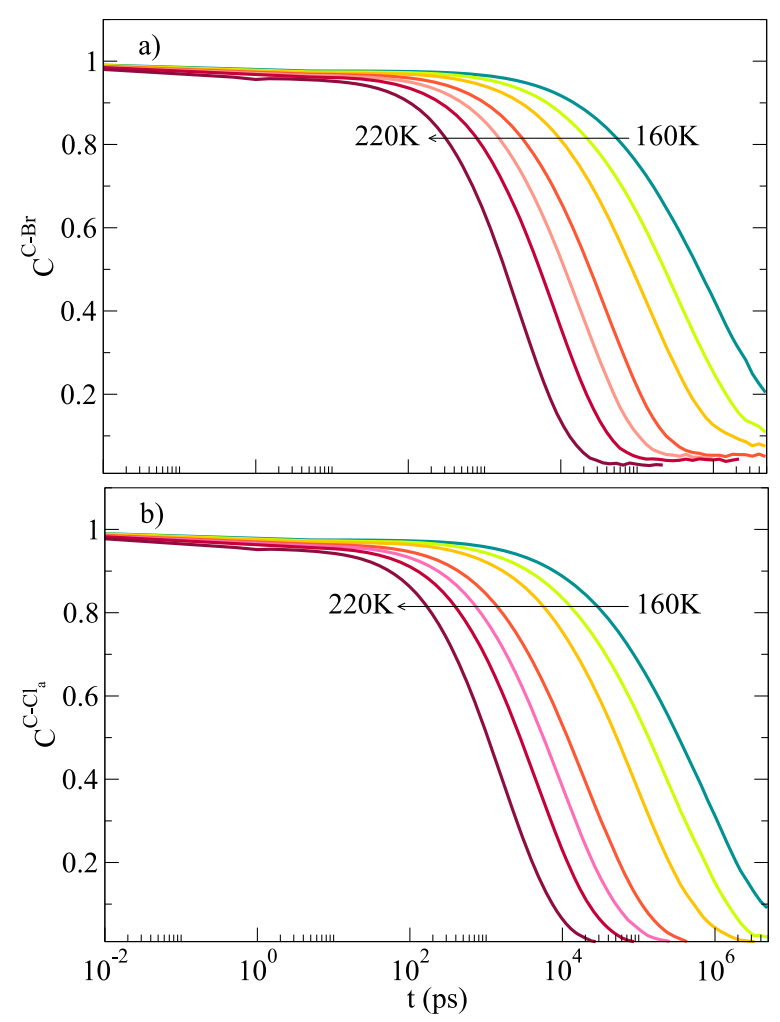

FIG. 1. Rotational correlation functions for all (a) $\mathrm{C}-\mathrm{Br}$ and (b) $\mathrm{C}-\mathrm{Cl}_{a}$ directions for temperatures ranging from $160 \mathrm{~K}$ to $220 \mathrm{~K}$ in steps of $10 \mathrm{~K}$.

by means of dielectric and NQR techniques ${ }^{27,28}$ and were also observed by us for $\mathrm{CCl}_{4},{ }^{31,48}$ where the same groups of molecules were classified as slow (I and IV) and fast (II and III).

We have calculated relaxation times associated with all bromine atoms in the system from self-correlation functions [Eq. (1)], since them can be directly compared with experimental results based on dielectric techniques, reported

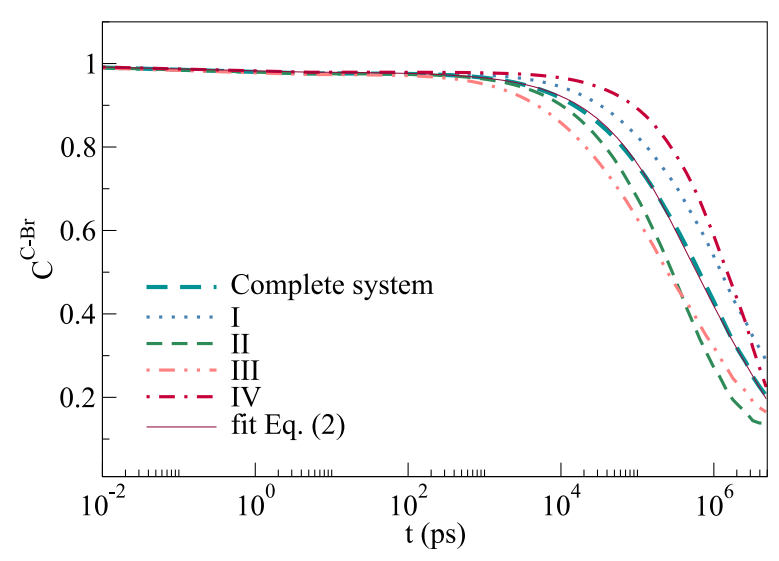

FIG. 2. Rotational correlation functions for C-Br bonds at $160 \mathrm{~K}$, calculated for molecules belonging to the four different groups in the system and for the whole system. The fit of the rotational correlation function for the complete system according to Eq. (2), with $C_{1}=0.68, \beta_{1}=0.54, C_{2}=0.3, \beta_{2}=0.72, \tau_{1}$ $=3.2 \times 10^{6} \mathrm{ps}$, and $\tau_{2}=2.4 \times 10^{5} \mathrm{ps}$, is also included. Correlation functions of groups I and IV are similar and the same holds for groups II and III (this behavior was observed for all studied temperatures). This coincidence among correlation functions is the first signature of two main dynamics: the faster one, where molecules of groups II and III are involved, and the slower one, involving molecules of groups I and IV. 
earlier $^{27}$ (because the $\mathrm{C}-\mathrm{Br}$ bond defines the molecular dipole direction). The contribution to the relaxation spectrum from $\alpha$ - and $\beta$-process may be deconvoluted using the William ansatz, ${ }^{45,53,54}$

$$
C(t)=C_{1} e^{\left(\frac{-t}{\tau_{1}}\right)^{\beta_{1}}}+C_{2} e^{\left(\frac{-t}{\tau_{2}}\right)^{\beta_{2}}} .
$$

The self-correlation functions for all $\mathrm{C}-\mathrm{Br}$ bonds in the system are well fitted with a combination of two stretched exponentials. The Kohlrausch-Williams-Watts (KWW) or stretched exponential decay function is the most commonly used empirical decay function for handling relaxation data affected by disorder. ${ }^{55}$ It is very successful in describing relaxation data of many disordered systems, since it is capable of capturing the large range of rate constants involved. We found, for the whole range of studied temperatures, $0.63 \leq C_{1} \leq 0.74$, $0.54 \leq \beta_{1} \leq 0.71$ (associated with the longer relaxation time $\tau_{1}$ ) and $0.24 \leq C_{2} \leq 0.35,0.72 \leq \beta_{2} \leq 0.8$ (associated with the shorter relaxation time $\tau_{2}$ ). Relaxation times $\tau_{1}$ and $\tau_{2}$, associated with all the bonds $\mathrm{C}-\mathrm{Br}$ in the system, showing a very good agreement with experimental results, are plotted in Fig. 3 along with dielectric and NQR results taken from Ref. 27. $\tau_{1}$ is compatible with $\tau_{\text {long }}$ and $\tau_{2}$ with $\tau_{\text {short }}$. Dielectric spectra for $\mathrm{CBrCl}_{3}$, as was reported earlier, ${ }^{28}$ are very well described by a Havriliak-Negami function for the $\alpha$-processes. From this function, $\beta_{D}$, equivalent to the stretching exponent in a decay behavior, is obtained. The stretching parameters $\beta_{2}$ obtained by fitting the self-correlation functions of $\mathrm{C}-\mathrm{Br}$ bonds are similar to $\beta_{D}$.

The molecular reorientation processes occurring during the simulations are sudden large-angle jumps of $\mathrm{Cl}$ and $\mathrm{Br}$ atoms. In Fig. 4, coordinates of $\mathrm{Br}$ and $\mathrm{Cl}$ atoms of one molecule arbitrarily chosen are shown as a function of time during $100 \mathrm{~ns}$. The $\mathrm{C}$ atom is in a lattice node, and the remaining atoms of the molecule exchange equilibrium positions with each other: two types of jumps are possible in this situation, one in which all atoms exchange their previous average position, i.e., four atoms' jump $\left(\mathrm{C}_{2}\right.$ jumps), and the other where an atom keeps its average position and the remaining three exchange positions between them $\left(\mathrm{C}_{3}\right.$ jumps $)$.

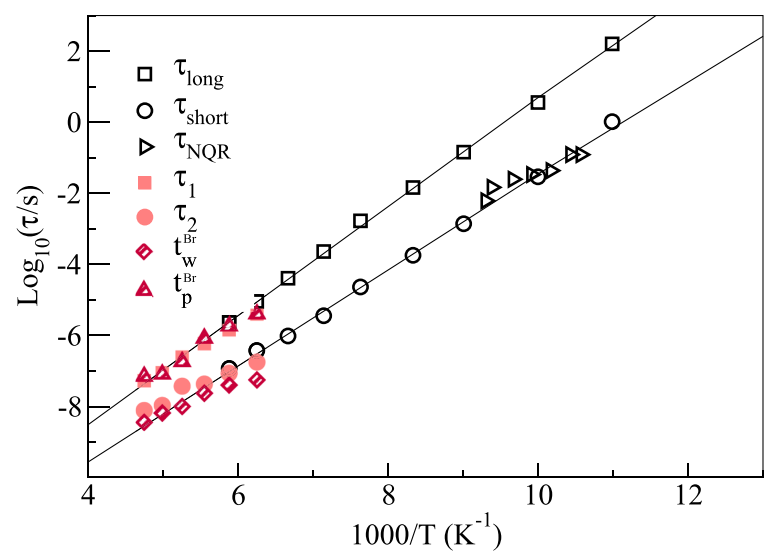

FIG. 3. Spin-lattice relaxation times obtained by molecular dynamics simulations from $\mathrm{C}-\mathrm{Br}$ self-correlation functions (full squares and circles). Waiting (diamonds) and persistence (triangles) mean times are also shown. $\tau_{\text {long, short }}$ (empty squares and circles) are relaxation times obtained by dielectric means for $\mathrm{CBrCl}_{3}$, taken from Ref. 27. Black straight lines are fits of $\tau_{\text {long }}$ and $\tau_{\text {short }}$. Results from NQR studies of $\mathrm{CBrCl}_{3}{ }^{27}$ (empty triangles) are also included.
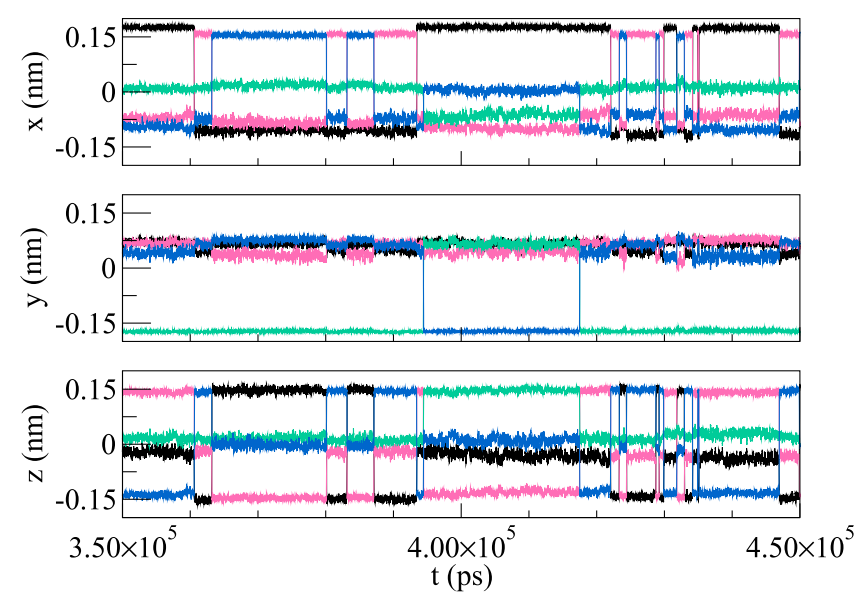

FIG. 4. $\mathrm{Cl}$ and $\mathrm{Br}$ coordinates relative to the $\mathrm{C}$ atom for one arbitrary molecule of group II at $160 \mathrm{~K}$. Black, pink, green, and blue are for $\mathrm{Br}, \mathrm{Cl}_{a}, \mathrm{Cl}_{b}$, and $\mathrm{Cl}_{c}$, respectively.

It is known that in the case of molecular liquids and hardsphere-like colloidal glasses, on approaching the glass transition to an amorphous solid-like state, the dynamics becomes intermittent and shows large spatiotemporal fluctuations, also known as dynamic heterogeneities. ${ }^{56,57}$ The dynamics is spatiotemporal heterogeneous since the probability that a particle rearranges in a given time interval is not spatially uniform, as long as the considered time interval is smaller than the relaxation time. Glassy dynamics is intermittent, as particles suddenly jump out of the cage formed by their neighbors, and heterogeneous, as these jumps are not uniformly distributed across the system. ${ }^{57,58}$ Since particle jumps are an ubiquitous feature of supercooled liquids, ${ }^{59}$ it is interesting to consider their role in an glass system in which only orientational dynamics can be frozen, as $\mathrm{CBrCl}_{3}$.

The jumps for all molecules were detected using a running test algorithm based on a signal-to-noise measure described in our previous work for $\mathrm{CCl}_{4}{ }^{48}$ After analyzing the trajectories for the $N=512$ molecules in the system, the times $t^{n, i}$ at which every single jump occurs were registered. The index $i$ indicates the $i$ th jump of the $n$ th-molecule, $n=1, \ldots$, $N$. The method of jump detection also allows us to distinguish which atoms of each molecule are involved in the jump process. A careful inspection confirmed that for all temperatures there were just a handful of cases corresponding to $C_{2}$ type rotations and therefore they were neglected in the analysis.

In Fig. 5, all registered times $t^{n, i}$ for $\mathrm{CBrCl}_{3}$ at $160 \mathrm{~K}$ are shown. The molecule number $n$ is such that molecules $n=1$, $\ldots, 128, n=129, \ldots, 256, n=257, \ldots, 384$, and $n=385, \ldots$, 512 belong to groups I, II, III, and IV, respectively. In order to compare with $\mathrm{CCl}_{4}$, the times at which jumps occur for this compound are also plotted at the same temperature, with data taken from our previous work. ${ }^{48}$

A feature of Fig. 5 is important to highlight: the distribution of jumps for $\mathrm{CCl}_{4}$ is uniform in time, while for $\mathrm{CBrCl}_{3}$ some correlations arise. Periods of fast and slow reorientations are noticeable, indicative of heterogeneous dynamics.

The statistics behind the rotational jumps can be evaluated by studying the waiting times between successive jumps. We 


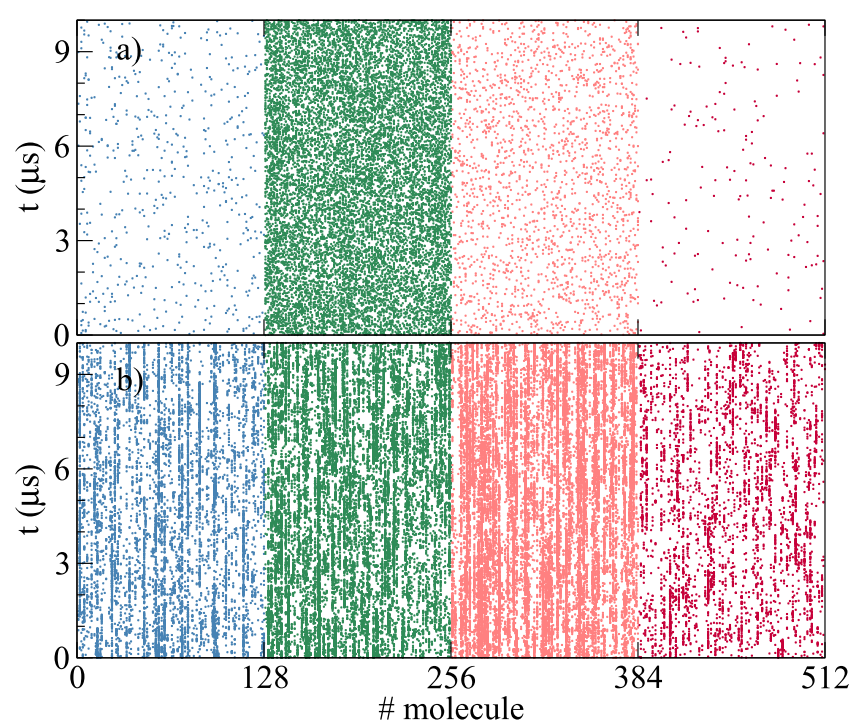

FIG. 5. Registered jumps for (a) $\mathrm{CCl}_{4}$ and (b) $\mathrm{CBrCl}_{3}$ for every molecule of the system during a simulation of $10 \mu$ s at $160 \mathrm{~K}$. Molecules numbered from 1 to 128,129 to 256,257 to 384 , and 381 to 512 belong to groups I, II, III, and IV, respectively. First 8 molecules of every group are in the same unit cell. Second 8 molecules are in another unit cell and so on. $\mathrm{CCl}_{4}$ shows a Poisson distribution of jumps as was highlighted before, ${ }^{48}$ but $\mathrm{CBrCl}_{3}$ moves away from this behavior. For the $\mathrm{CCl}_{4}$ system, a clear difference in the number of jumps among groups arises, while in the $\mathrm{CBrCl}_{3}$ system, there is no such clear distinction. In the $\mathrm{CBrCl}_{3}$ system, it is worth noticing that molecules with short waiting time between jumps are more probable to have a next short waiting time.

define the waiting time $t_{w}^{n, i}$ as the time between two consecutive jumps of the same molecule, i.e., $t_{w}^{n, i}=t^{n, i+1}-t^{n, i}$. In Fig. 6 , we show the distribution of waiting times for rotational events for $\mathrm{CBrCl}_{3}$ and $\mathrm{CCl}_{4}$ directly obtained from the simulated trajectories. For $\mathrm{CCl}_{4}$, the waiting time distribution is a Poisson process, following an exponential law $\sim e^{t / \tau_{w}}$. Calculating the
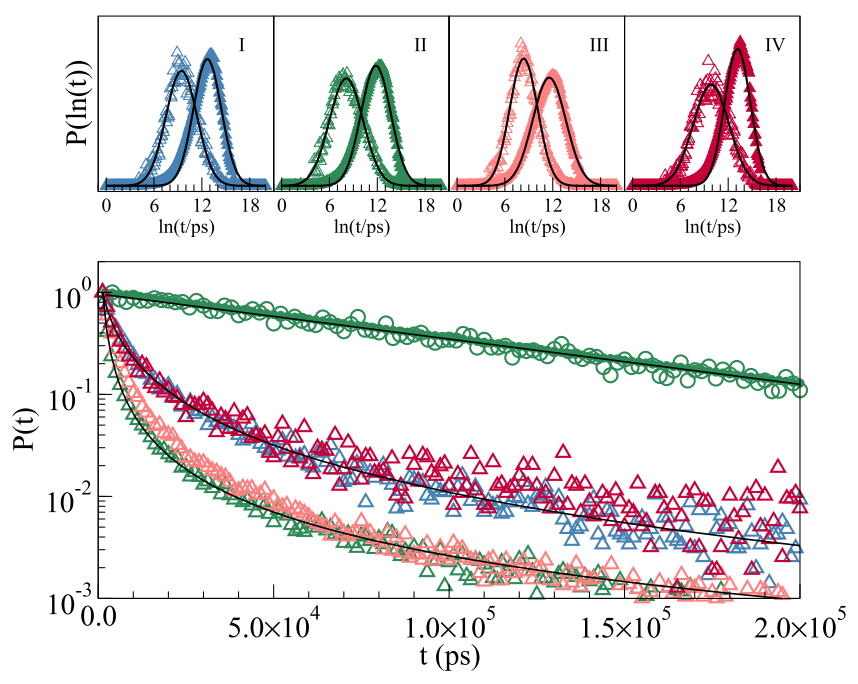

FIG. 6. Probability distributions of waiting times (empty triangles) for groups I (blue), II (green), III (pink), and IV (red) of $\mathrm{CBrCl}_{3}$. Probability distributions of waiting times (empty circles) and persistence times (full circles) for group II of $\mathrm{CCl}_{4}$ are also included. In the top figures, the probability distributions of the natural logarithm of waiting times (empty triangles) and persistence times (full triangles) for $\mathrm{CBrCl}_{3}$ are plotted. All data are at $160 \mathrm{~K}$. Black solid lines are curves resulting from fitting the data with a normal function for the four top figures, an exponential function for $\mathrm{CCl}_{4}$, and a log-normal function for $\mathrm{CBrCl}_{3}$ (just fits of groups I and II are shown for $\mathrm{CBrCl}_{3}$ ). probability distribution of $x=\ln \left(t_{w}\right)$, a normal distribution, $\sim e^{\frac{(x-\mu)^{2}}{2 \sigma^{2}}}$, results suitable to fit the data in the case of $\mathrm{CBrCl}_{3}$. A standard variable change procedure gives a log-normal distribution for the probabilities as a function of time, with mean equal to $\tau_{w}=e^{\mu+\frac{\sigma^{2}}{2}}$.

The dependence of one waiting time $\left(t_{w}^{n, i}\right)$ on the previous waiting time $\left(t_{w}^{n, i-1}\right)$ provides a measure for the heterogeneities' lifetime. ${ }^{60}$ In the case of homogeneous dynamics for one molecule, subsequent waiting times are uncorrelated. For very long heterogeneity lifetimes, a long waiting time is most likely to be followed by another long waiting time, while a short waiting time is most likely followed by another short waiting time. In order to analyze correlations between subsequent waiting times, we have calculated the contour plots shown in Fig. 7. These contour plots are constructed from the probability distribution of having $t_{w}^{n, i}$ considering that the previous waiting time was $t_{w}^{n, i-1}$. The analysis was made for the $\mathrm{CBrCl}_{3}$ and $\mathrm{CCl}_{4}$ systems and is shown in Fig. 7, at the highest and lowest simulated temperatures. In the $\mathrm{CBrCl}_{3}$ case, at $160 \mathrm{~K}$, an elongated cloud along the diagonal leads to a correlation of $r=0.52$ between the logarithm of subsequent waiting times; while for the highest simulated temperature, $220 \mathrm{~K}$, this value is reduced to 0.25 , almost half of the previous case, closer to the $\mathrm{CCl}_{4}$ case, where correlations are 0.22 and 0.23 at $160 \mathrm{~K}$ and $220 \mathrm{~K}$, respectively.

One consequence of correlations among waiting times is that dynamical processes can be partitioned according to whether they coincide with waiting events (successive jumps of the same molecule) or persistence events (the time from the molecule initial condition before it jumps). The distinctions between these two classes of processes are responsible for a host of nonlinear phenomena that are characteristic of deeply supercooled liquids. ${ }^{61}$

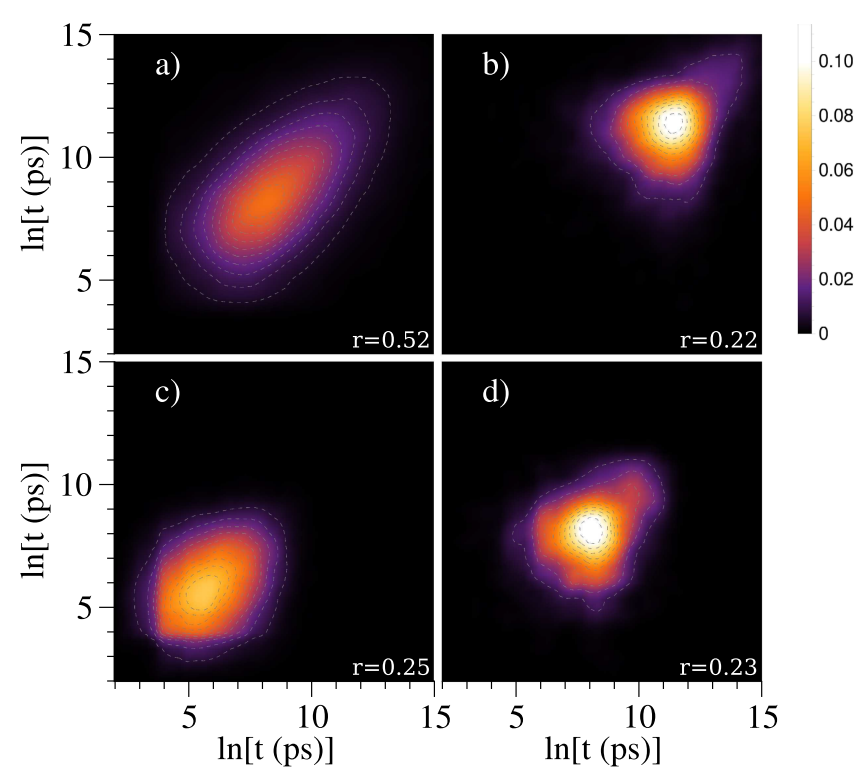

FIG. 7. Probability distribution functions and contour plots (dashed lines) showing the dependence between subsequent waiting times for all molecules in a system of (a) $\mathrm{CBrCl}_{3}$ molecules at $160 \mathrm{~K}$, (b) $\mathrm{CCl}_{4}$ molecules at $160 \mathrm{~K}$, (c) $\mathrm{CBrCl}_{3}$ molecules at $220 \mathrm{~K}$, and (d) $\mathrm{CCl}_{4}$ molecules at $220 \mathrm{~K}$. Vertical axis and horizontal axis indicate waiting times and previous waiting time, respectively. 
The distribution of times characterizing the molecular intermittent behavior can be viewed in terms of the persistence time, defined as the time from any given point in the trajectory of a single molecule $t_{0}^{i}$, until the next jump event $t^{n, j}, t_{p}^{n, i}=t^{n, j}-t_{0}^{i}$, and the time between two jumps (the, defined before, waiting time). For uncorrelated sequence of events, persistence times and waiting times have the same distribution, and the probability that an event has not occurred in a time $t$ is $e^{-t / \tau}$, with $\tau=t_{w}=t_{p}$, were $t_{w}$ and $t_{p}$ are mean waiting and persistence times, respectively. Such intermittencies are known as Poisson processes. In the case of glassy dynamics, however, statistics of events is far from Poissonian because an event becomes more likely when a similar event has occurred more recently. As a consequence, when dynamics are correlated, waiting times are typically shorter than persistence times. ${ }^{61,62}$

We have calculated persistence time distributions for $\mathrm{CBrCl}_{3}$ and $\mathrm{CCl}_{4}$, for all simulated temperatures and for the four groups of nonequivalent molecules. We found that for $\mathrm{CBrCl}_{3}$ persistence and waiting times, distributions decouple (see Fig. 6). For $\mathrm{CCl}_{4}$, the situation is different, persistence and waiting times are equally distributed and Poissonian. Mean values $\tau_{w}$ and $\tau_{p}$ for each group in $\mathrm{CBrCl}_{3}$ and $\mathrm{CCl}_{4}$ are shown in Fig. 8. In the $\mathrm{CCl}_{4}$ case, an impressive coincidence among waiting and persistence mean times arises, emphasizing the Poissonian behavior of the system. For $\mathrm{CBrCl}_{3}$, the
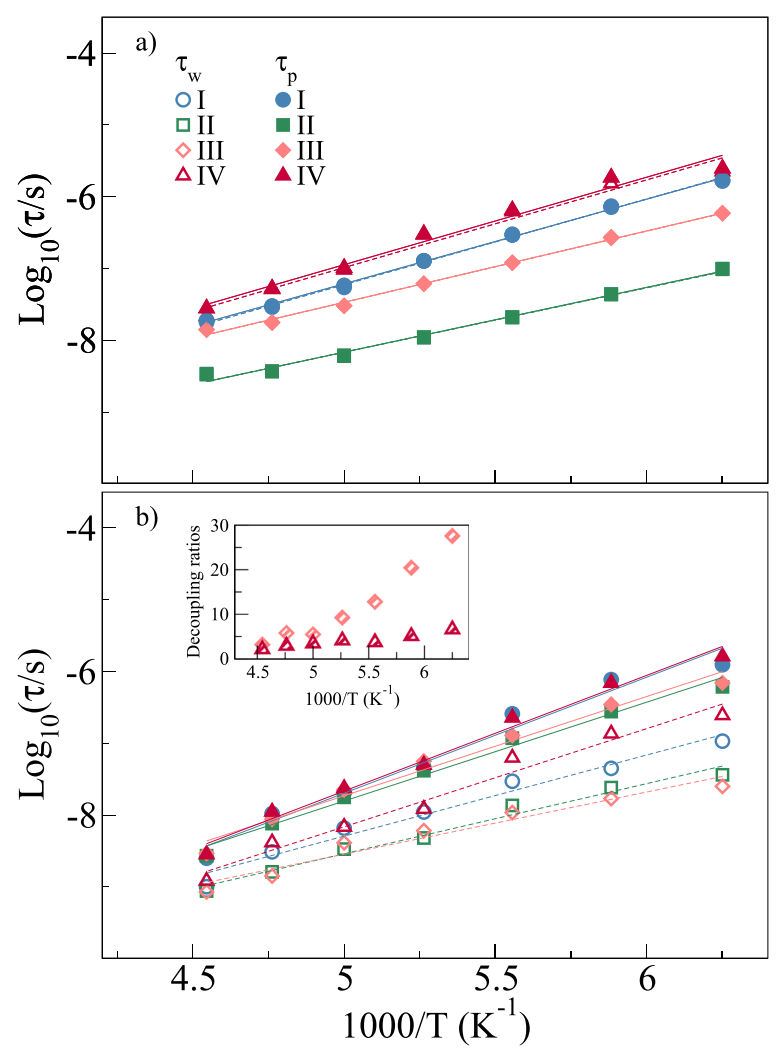

FIG. 8. Mean waiting (empty symbols) and persistence (full symbols) times obtained from probability distributions as a function of temperature for the four groups (I, II, III, and IV) in the system. Results for $\mathrm{CCl}_{4}$ in (a) show coincidence among mean waiting and persistence times for molecules in the same group. In case (b) for $\mathrm{CBrCl}_{3}$, persistence mean times are longer than waiting mean times. In the inset of (b) decoupling ratios, calculated as $t_{p} / t_{w}$ are shown for groups III (diamonds) and IV (triangles). correlation among jump processes is responsible for the marked difference between waiting and persistence mean times, where mean waiting times are considerably shorter than mean persistence times. In Fig. 8, we also show the decoupling ratios, defined as $\tau_{p} / \tau_{w}$, for groups III and IV. For the higher studied temperatures, the ratio is close to one, as expected for standard liquid conditions, ${ }^{61}$ but for lower temperatures the ratio grows, as in the fluctuation dominated regime of supercooled liquids, where this is a signature of the breakdown of Stokes-Einstein relations. ${ }^{62,63}$

Persistence times, defined as the average waiting time from any given point in the trajectory of a single molecule, until the next jump event, are comparable to the structural relaxation time $t_{\alpha}$ in simple models of glass dynamics. ${ }^{64}$ The probability distribution of persistence times is comparable to typical results of bulk experiments, due to the time-weighted nature of typical observations. Since available experimental times describing $\mathrm{CBrCl}_{3}$ dynamics are the result from dielectric measurements, where the bromine atom is sensed, we also calculated persistence and waiting mean times for all bromine atoms in the simulated system.

We define the waiting time for a bromine atom as the difference in time between successive jumps of a bromine atom belonging to one molecule of the system. The probability distributions for waiting times of all bromine atoms in the system, as was the case for all molecular jumps, also follow a log-normal distribution for all simulated temperatures. The same behavior was observed for squared waiting times. From mean values of these distributions, we calculated $\tau_{w}^{B r}$ and $\tau_{p}^{B r}$, mean waiting and persistence times for all bromine atoms in the system. These results are plotted in Fig. 3. Persistence mean times of bromine atoms show an impressive good agreement with times $\tau_{\text {long }}$ obtained from dielectric measurements, and waiting mean times coincide with $\tau_{\text {short }}$ obtained from the same experiment. The interpretation of this coincidence is that $\alpha$-relaxation is associated with times in which bromine atoms are not reorienting, but persisting in one position, and $\beta$-relaxation is associated with the fastest process in which the molecules are jumping and reorienting.

\section{CONCLUSIONS}

We performed extensive molecular dynamics simulations of $\mathrm{CBrCl}_{3}$ in the monoclinic low-temperature phase. By studying time reorientational auto-correlation functions as a function of temperature, we found distinct behaviors for $\mathrm{C}-\mathrm{Br}$ bonds when comparing with the other $\mathrm{C}-\mathrm{Cl}$ bonds in the molecule. We found two characteristic times emerging from these functions: a longer one, compatible with $\tau_{\text {long }}$ associated with $\alpha$-relaxation, and a shorter one, compatible with $\tau_{\text {short }}$ associated with $\beta$-relaxation from dielectric measurements.

$\mathrm{CBrCl}_{3}$ dynamics reorientations are due to sudden jumps between equivalent positions of the atoms. By computing jumping times directly from simulation trajectories, we calculated waiting and persistence times. Probability distributions of waiting and persistence times show a log-normal behavior, from which is possible to obtain mean values for waiting and persistence times. 
We calculated mean waiting and persistence times, first distinguishing among groups of molecules and second for all bromine atoms in the system. From the groups' analysis, we found that different groups have different characteristic mean times, and when comparing mean waiting and persistence times, a difference arises, showing correlations in the system dynamics. From the bromine atoms' analysis, we found that mean waiting times are compatible with the shorter characteristic time, associated with $\beta$-relaxation and mean persistence times are compatible with the larger characteristic time, associated with $\alpha$-relaxation.

Although groups of different molecules are well defined for $\mathrm{CCl}_{4}$, where every molecule belonging to a group has the same molecular environment, in $\mathrm{CBrCl}_{3}$, where this assertion is no longer valid due to the disorder introduced by the bromine atom, groups of molecules are still well defined for this compound. Molecules belonging to different groups of the $\mathrm{CBrCl}_{3}$ system experiment different dynamics, as can be observed when studying autocorrelation functions for bonds of molecules belonging to different groups or when the mean persistence and waiting times are calculated.

To our knowledge, this is the first time that an analysis in terms of microscopic molecular jumps is done over an orientational low-dimensional glass. We showed that the orientational glass former $\mathrm{CBrCl}_{3}$ has all the major features of structural glass formers, without of course the structural degree of freedom.

\section{ACKNOWLEDGMENTS}

N.B.C., M.Z., and P.S. acknowledge financial support of SECYTUNC and CONICET.

This work used computational resources from CCAD Universidad Nacional de Córdoba (http://ccad.unc.edu.ar/), in particular the Mendieta Cluster, which is part of SNCAD MinCyT, República Argentina.

This work was partially supported by the Spanish Ministry of Economy and Competitiveness through Project No. FIS2014-54734-P.

${ }^{1}$ C. Josserand, A. V. Tkachenko, D. M. Mueth, and H. M. Jaeger, Phys. Rev. Lett. 85, 3632 (2000).

${ }^{2}$ P. E. Anderson, H. J. Jensen, L. Oliveira, and P. Sibani, Complexity 10, 49 (2004).

${ }^{3}$ S. Bustingorry, L. F. Cugliandolo, and J. L. Iguain, J. Stat. Mech.: Theory Exp. 2007, P09008.

${ }^{4}$ J. Gorchon, S. Bustingorry, J. Ferré, V. Jeudy, A. Kolton, and T. Giamarchi, Phys. Rev. Lett. 113, 027205 (2014).

${ }^{5}$ C. A. Angell, Science 267, 1924 (1995).

${ }^{6}$ R. Schilling, J. Phys.: Condens. Matter 12, 6311 (2000).

${ }^{7}$ M. Ricker and R. Schilling, Phys. Rev. E 72, 011508 (2005).

${ }^{8}$ F. Ritort and P. Sollich, Adv. Phys. 52, 219 (2003).

${ }^{9}$ A. Vispa, M. Romanini, M. A. Ramos, L. C. Pardo, F. J. Bermejo, M. Hassaine, A. I. Krivchikov, J. W. Taylor, and J. L. Tamarit, Phys. Rev. Lett. 118, 105701 (2017).

${ }^{10}$ H. Suga and S. Seki, J. Non-Cryst. Solids 16, 171 (1974).

${ }^{11}$ R. Puertas, M. A. Rute, J. Salud, D. O. López, S. Diez, J. K. van Miltenburg, L. C. Pardo, J. L. Tamarit, M. Barrio, M. A. Pérez-Jubindo, and M. R. de la Fuente, Phys. Rev. B 69, 224202 (2004).

${ }^{12}$ M. Romanini, P. Negrier, J. L. Tamarit, S. Capaccioli, M. Barrio, L. Pardo, and D. Mondieig, Phys. Rev. B 85, 134201 (2012).

${ }^{13}$ G. A. Vdovichenko, A. I. Krivchikov, O. Korolyuk, J. L. Tamarit, L. Pardo, M. Rovira-Esteva, F. J. Bermejo, M. Hassaine, and M. Ramos, J. Chem. Phys. 143, 084510 (2015).
${ }^{14}$ S. C. Pérez, M. Zuriaga, P. Serra, A. Wolfenson, P. Negrier, and J. L. Tamarit, J. Chem. Phys. 143, 134502 (2015).

${ }^{15}$ B. Ben Hassine, P. Negrier, M. Romanini, M. Barrio, R. Macovez, A. Kallel, D. Mondieig, and J. L. Tamarit, Phys. Chem. Chem. Phys. 18, 10924 (2016).

${ }^{16}$ M. Romanini, M. Barrio, S. Capaccioli, R. Macovez, M. D. Ruiz-Martin, and J. L. Tamarit, J. Phys. Chem. C 120, 10614 (2016).

${ }^{17}$ P. Tripathi, E. Mitsari, M. Romanini, P. Serra, J. L. Tamarit, M. Zuriaga, and R. Macovez, J. Chem. Phys. 144, 164505 (2016).

${ }^{18}$ C. A. Angell, Curr. Opin. Solid State Mater. Sci. 1, 578 (1996).

${ }^{19}$ L. Pardo, M. Barrio, J. L. Tamarit, D. Lopez, J. Salud, P. Negrier, and D. Mondieig, Phys. Chem. Chem. Phys. 13, 2644-2649 (2001).

${ }^{20}$ B. Parat, L. C. Pardo, M. Barrio, J. L. Tamarit, P. Negrier, J. Salud, D. O. López, and D. Mondieig, Chem. Mater. 17, 3359 (2005).

${ }^{21}$ M. Barrio, J. L. Tamarit, P. Negrier, L. C. Pardo, N. Veglio, and D. Mondieig, New J. Chem. 32, 232 (2008).

${ }^{22}$ L. Pardo, M. Barrio, J. L. Tamarit, D. López, J. Salud, P. Negrier, and D. Mondieig, Chem. Phys. Lett. 308, 204210 (1999).

${ }^{23}$ L. Pardo, M. Barrio, J. L. Tamarit, D. López, J. Salud, P. Negrier, and D. Mondieig, Chem. Phys. Lett. 321, 438 (2000).

${ }^{24}$ L. Pardo, M. Barrio, J. L. Tamarit, P. Negrier, D. López, J. Salud, and D. Mondieig, J. Phys. Chem. B 105, 10326-10334 (2001).

${ }^{25}$ S. Pothoczki, A. Ottochian, M. Rovira-Esteva, L. Pardo, J. L. Tamarit, and G. Cuello, Phys. Rev. B 85, 014202 (2012).

${ }^{26}$ T. Ohta, O. Yamamuro, and T. Matsuo, J. Phys. Chem. 99, 2403 (1995).

${ }^{27}$ M. Zuriaga, L. Pardo, P. Lunkenheimer, J. L. Tamarit, N. Veglio, M. Barrio, F. J. Bermejo, and A. Loidl, Phys. Rev. Lett. 103, 75701 (2009).

${ }^{28}$ M. J. Zuriaga, S. C. Perez, L. C. Pardo, and J. L. Tamarit, J. Chem. Phys. 137, 054506 (2012).

${ }^{29}$ S. Lee-Dadswell, B. Torrie, O. Binbrek, and B. Powell, Phys. B 241, 459 (1997).

${ }^{30}$ O. Binbrek, S. E. Lee-Dadswell, B. Torrie, and B. M. Powell, Mol. Phys. 96, 785 (1999).

${ }^{31}$ M. Zuriaga, M. Carignano, and P. Serra, J. Chem. Phys. 135, 044504 (2011).

${ }^{32}$ P. Lunkenheimer and A. Loidl, Chem. Phys. 284, 205 (2002).

${ }^{33}$ G. P. Johari and M. Goldstein, J. Chem. Phys. 53, 2372 (1970).

${ }^{34}$ G. Johari, J. Chem. Phys. 58, 1766-1770 (1973).

${ }^{35}$ K. Ngai, Phys. Rev. E 57, 7346-7349 (1998).

${ }^{36}$ M. Jimenez-Ruiz, A. Criado, F. J. Bermejo, G. J. Cuello, F. R. Trouw, R. Fernandez-Perea, H. Löwen, C. Cabrillo, and H. E. Fischer, Phys. Rev. Lett. 83, 2757 (1999).

${ }^{37}$ M. Jiménez-Ruiz, M. A. González, F. Bermejo, M. A. Miller, N. O. Birge, I. Cendoya, and A. Alegría, Phys. Rev. B 59, 9155 (1999).

${ }^{38}$ U. Schneider, R. Brand, P. Lunkenheimer, and A. Loidl, Phys. Rev. Lett. 84, 5560-5563 (2000).

${ }^{39}$ G. Johari, G. Power, and J. Vij, J. Chem. Phys. 116, 5908-5909 (2002).

${ }^{40}$ K. Ngai and M. Paluch, J. Chem. Phys. 120, 857 (2004).

${ }^{41}$ F. Affouard, E. Cochin, R. Decressain, and M. Descamps, J. Chem. Phys. 123, 084501 (2005).

${ }^{42}$ L. Pardo, P. Lunkenheimer, and A. Loidl, J. Chem. Phys. 124, 124911 (2006).

${ }^{43}$ S. Capaccioli, K. Ngai, and N. Shinyashiki, J. Chem. Phys. B 111, 8197 (2007).

${ }^{44}$ S. Capaccioli and K. Ngai, J. Chem. Phys. 135, 104504 (2011).

${ }^{45}$ D. Fragiadakis and C. Roland, Phys. Rev. E 95, 022607 (2017).

${ }^{46}$ M. Voguel and E. Rossler, J. Phys. Chem. B 104, 4285 (2000).

${ }^{47}$ M. Voguel and E. Rossler, J. Chem. Phys. 114, 5802 (2001).

${ }^{48}$ N. B. Caballero, M. Zuriaga, M. Carignano, and P. Serra, J. Phys. Chem. B 120, 860 (2016).

${ }^{49}$ N. Caballero, M. Zuriaga, M. Carignano, and P. Serra, J. Chem. Phys. 136, 094515 (2012).

${ }^{50}$ S. Cohen, R. Powers, and R. Rudman, Acta Crystallogr., Sect. B: Struct. Crystallogr. Cryst. Chem. B35, 1670 (1979).

${ }^{51}$ P. Negrier, J. L. Tamarit, M. Barrio, L. C. Pardo, and D. Mondieig, Chem. Phys. 336, 150 (2007).

${ }^{52}$ S. Kämmerer, W. Kob, and R. Schilling, Phys. Rev. E 56, 5450 (1997).

${ }^{53} \mathrm{G}$. Williams, Electric Phenomena in Polymer Science (Springer, 1979), Vol. 59.

${ }^{54}$ D. Fragiadakis and C. Roland, Phys. Rev. E 89, 052304 (2014).

${ }^{55}$ R. Richert and A. E. Blumen, Disorder Effects on Relaxational Processes: Glasses, Polymers, Proteins (Springer Science \& Business Media, 1994).

${ }^{56}$ R. Pastore, M. P. Ciamarra, G. Pesce, and A. Sasso, Soft Matter 11, 622 (2014). 
${ }^{57}$ R. Pastore, G. Pesce, A. Sasso, and M. Pica Ciamarra, J. Phys. Chem. Lett. 8, 1562 (2017).

${ }^{58}$ R. Pastore, A. Coniglio, and M. P. Ciamarra, Sci. Rep. 5, 11770 (2015).

${ }^{59}$ M. P. Ciamarra, R. Pastore, and A. Coniglio, Soft Matter 12, 358 (2016).

${ }^{60}$ D. Bingemann, R. M. Allen, and S. W. Olesen, J. Chem. Phys. 134, 024513 (2011).
${ }^{61}$ D. Chandler and J. P. Garrahan, Annu. Rev. Phys. Chem. 61, 191 (2010). ${ }^{62}$ L. O. Hedges, L. Maibaum, D. Chandler, and J. P. Garrahan, J. Chem. Phys. 127, 211101 (2007).

${ }^{63}$ Y. Jung, J. P. Garrahan, and D. Chandler, Phys. Rev. E 69, 061205 (2004).

${ }^{64}$ J. W. Ahn, B. Falahee, C. D. Piccolo, M. Vogel, and D. Bingemann, J. Chem. Phys. 138, 12A527 (2013). 